\title{
Research report \\ Human large-scale oscillatory brain activity during an operant shaping procedure
}

\author{
Andreas Keil ${ }^{\mathrm{a}, *}$, Matthias M. Müller ${ }^{\mathrm{b}}$, Thomas Gruber $^{\mathrm{b}}$, Christian Wienbruch ${ }^{\mathrm{a}}$, \\ Thomas Elbert ${ }^{\mathrm{a}}$ \\ ${ }^{a}$ Department of Psychology, University of Konstanz, Box D-25, D-78434 Konstanz, Germany \\ ${ }^{\mathrm{b}}$ Cognitive Neuroscience, Department of Psychology, University of Liverpool, Liverpool, UK
}

\begin{abstract}
The present study aimed at examining the oscillatory brain-electric correlates of human operant learning using high-density electroencephalography (EEG). Induced gamma-band activity (GBA) was studied using a fixed-interval reinforcement schedule with a variable limited hold period, which was decreased depending on response accuracy. Thus, participants' behavior was shaped during the course of the learning session. After each response, numbers indicating the money value of that response served as reinforcing stimuli. Random reinforcement and self-paced button pressing without reinforcement were added as control conditions. GBA around $40 \mathrm{~Hz}$ was enhanced at posterior electrodes in response to visual feedback stimuli during shaping and random reward compared to the self-paced pressing condition where no visual feedback was provided. Furthermore, shaping was associated with a pronounced left frontal lower gamma $(20-30 \mathrm{~Hz})$ increase in response to feedback stimuli, whereas this pattern was not observed in the random reinforcement and self-paced pressing conditions. The present findings are in line with the notion that macroscopic high-frequency dynamics of neuronal cell assemblies may be regarded as a mechanism involved in learning and memory formation.
\end{abstract}

Theme: Neural basis of behavior

Topic: Learning and memory: systems and functions

Keywords: Operant learning; Oscillatory brain activity; Memory formation

\section{Introduction}

Operant learning of contingencies between individual behavior and external reinforcers has been viewed as an important element of human behavior, as well as a valid and practicable laboratory model for learning per se $[6,44]$. Current theories of plasticity and learning describe cortical mechanisms on the system and neuronal level. On system level, several studies have demonstrated a relation between dorsolateral, medial and orbital pre-frontal cortex and acquisition of reinforcement contingencies in animals using electrophysiological [42] or lesion techniques $[32,33]$. Thus, frontal cortex appears to be part of a 891.

*Corresponding author. Tel.: +49-7531-884-003; fax: +49-7531-882-

E-mail address: andreas.keil@uni-konstanz.de (A. Keil). widespread network that is involved in learning new behaviors and includes a variety of other structures such as the amygdala, hippocampus, hypothalamus and sensory cortices $[13,23]$. Given these data, the question arises as to the mechanisms that act to integrate the neuronal activity within and between the elements of such a network.

Based on Hebb's proposal of the formation of cell assemblies by temporally correlated action potentials as the neuronal mechanisms underlying learning [16], recent theoretical proposals extended Hebb's model by the dynamical dimension of synchronized neuronal firing rates (e.g. Refs. [25,45,50]). High-frequency neuronal activity can be recorded from humans using traditional electrocortical measures [24] such as the electroencephalogram (EEG). In particular, induced gamma band activity $(\mathrm{GBA}>20 \mathrm{~Hz}$ ), which is not phase-locked to the onset of a stimulus, has attracted attention since it has been shown to 
correlate with formation and activation of object representations [47]. Recent work in animals [8], humans [26], and computational models [17] points to a possible role of high-frequency oscillatory brain activity in the acquisition of learned contingencies. Therefore, an increase of GBA in the human EEG may represent a signature of associative learning, reflecting oscillatory activity in neuronal assemblies [1,38]. On the other hand, functional imaging studies in humans and animal electrophysiology have shown that the amplitudes of responses to visual objects decrease in the course of learning [7], a fact that is commonly referred to as repetition suppression. Repetition suppression has been studied in the visual domain and has been related to higher-order visual processes such as associated with inferior temporal cortex [2]. This phenomenon has been attributed to an increase in the connectivity between the task-relevant brain areas and is thus not explained by habituation [4]. Alternatively, fronto-temporal connections may modulate visual processing according to information provided by memory systems [7]. Time-frequency analyses of electrophysiological data may be a useful tool in investigating these mechanisms because of their relation to neuronal rather than metabolic processes and their better time resolution, compared to blood flow measures [47]. Furthermore, changes in GBA power can be related to alterations in the local response amplitude and local coupling of neuronal assemblies [9,18]. Likewise, the spatio-temporal patterns of neuronal mass activity are expected to change in response to changes of reinforcement contingencies [10].

In the current study, we used a shaping paradigm that allowed keeping the participants in a state of ongoing learning according to an operant reinforcement schedule. Random (non-contingent) reinforcement and self-paced responding without reward served as control conditions. If the amplitude of the visual response is reduced in the course of the learning condition, decreased GBA amplitude in the visual cortex should be observed as a function of stimulus repetition specifically in this condition. In addition, frontal sites should show a GBA increase as a function of ongoing learning, reflecting changes in spatiotemporal activity that may be related to top-down modulation of visual processes by reorganizing memory systems.

\section{Materials and methods}

\subsection{Subjects}

Eleven healthy, right-handed university students (eight male, three female) received class credits or a small financial bonus for participation. Their age ranged from 19 to 33 years (mean age 23.2 years).

\subsection{Procedure and design}

After giving informed consent, participants were told that they would be given the opportunity to earn additional money by pressing a response key in two different tasks. Subsequently, the feedback stimuli indicating the actual amount or reward were explained. No further instructions were given concerning operant behavior and reward contingencies. The electrode array was applied to the subject's head and the participant entered the electrically shielded chamber where $\mathrm{s} /$ he was seated in a comfortable chair. Three test trials were conducted to ensure that the subject was able to understand the instructions and perform the task. After the test trials, three experimental blocks were conducted: (1) a block establishing a fixed interval reinforcement schedule with a variable limited hold period, referred to as 'shaping condition'; (2) a block without contingent reinforcement ('random reinforcement condition'); (3) a block of self-paced key pressing, referred to as 'self-paced condition'. The order of the learning vs. nonlearning conditions was counterbalanced across subjects.

\subsubsection{Shaping condition}

A fixed interval reinforcement schedule with a variable limited hold period was used for the operant control of the subjects' key presses in the shaping trials. The delay of the key press (i.e. onset of key press in relation to trial onset) was used to define the operant response rather than intensity or duration of the movement. This was done to keep the response itself easy and thus allow for the application of a shaping regime. Accordingly, the key was a simple switch not being sensitive to touch intensity or duration. Each shaping trial began with a cue stimulus (i.e. a brief color change of the fixation point). Reward was available only after an interval of $3 \mathrm{~s}$ after the cue, with an initial limited hold period of $900 \mathrm{~ms}$ at the outset of the shaping block (see Fig. 1, top). Thus, in the first trial, responses would be rewarded when being located between 3000 and $3900 \mathrm{~ms}$ after the initial cue. Subjects received a maximum amount of money if their response latency was exactly centered between $3 \mathrm{~s}$ and the actual limited hold duration, with increasing discrepancy being associated with less reinforcement. Accordingly, in the first trial, a response at $3450 \mathrm{~ms}$ would lead to maximum reward (i.e. $0.06 \mathrm{DM}$, see below). Temporal distance from the center of the reward interval resulted in less reward, according to a linear function declining in steps of 0.01 DM (see Fig. 1, top). The limited hold period was reduced by $100 \mathrm{~ms}$ when the subject obtained more than two consecutive maximal rewards. Thus, the optimal response latency was not constant throughout trials, but was subject to shaping, approaching a latency of $3000 \mathrm{~ms}$. Each rewarded key press immediately elicited feedback of the occurrence and amount of reward via a computer screen, followed by a 1500-ms interval after which a new trial began. Feedback stimuli were numbers ranging from ' 00 ' to ' 60 ', subtending a visual angle of $3^{\circ}$, with $500-\mathrm{ms}$ duration. Participants knew that a feedback of ' 60 ' meant additional $0.06 \mathrm{DM}$ reward, '30' was equivalent to $0.03 \mathrm{DM}$, etc. Key pressing outside the reward window resulted in no feedback at all. 

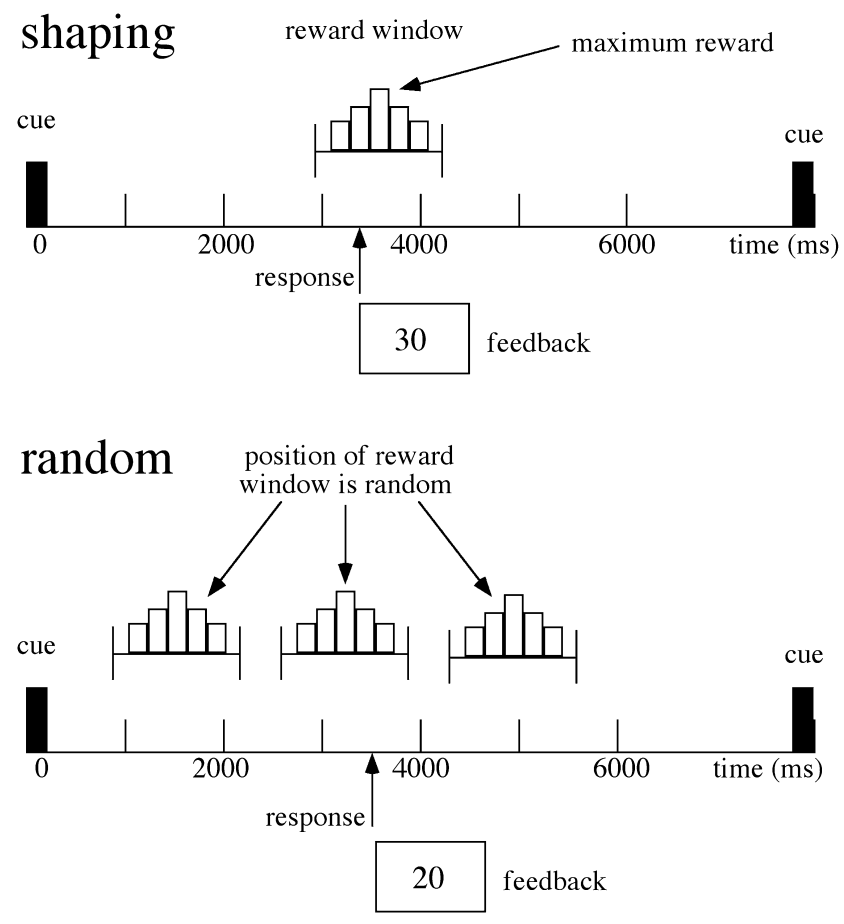

Fig. 1. Graphical representation of the experimental conditions 'shaping' and 'random reward'. Shaping (top): the size of the reward window was reduced in the course of the experiment, depending on participants' response (see Section 2). Random reinforcement (bottom): the reward window is shifted randomly from trial to trial. Non-contingent reward is provided.

If the subjects did not respond until $6000 \mathrm{~ms}$ after the cue, the trial was aborted and a zero reward was displayed on the screen. Only those trials that were rewarded with 30 or more points were included in the EEG analyses, in order to exclude trials with multiple key presses and those that reflected erratic key pressing.

\subsubsection{Random reinforcement condition}

As a control condition, trials were executed that involved key presses and occasional reward, but did not allow for operant learning. In these control trials, no contingent reinforcement, but randomized reward was given independently of the response interval (see Fig. 1, bottom). Occasional but randomized reinforcement was thought to keep motivation and attention on a comparable level as in the shaping condition, without involving modification of behavior in response to a reinforcement schedule. Given this reinforcement procedure, less rewarded responses could be expected than in the shaping condition. Since only trials with rewards of $\geq 30$ points were used for EEG analyses, this block included 180 trials. This was done to provide comparable amounts of trials resulting from all experimental conditions. Length of trials and type of feedback stimuli were identical for shaping and random reinforcement conditions.

\subsubsection{Self-paced condition}

As a further control of movement-related time-fre- quency modulations in the EEG, subjects were asked to press the key regularly in long intervals in order to control for the brain correlates planned key pressing. No feedback on performance was provided via the screen. A total of 100 trials were obtained in this condition.

\subsection{Electrophysiological recordings}

EEG recordings were made using an EGI 128-channel system (Electrical Geodesics, 1998) with a sampling rate of $500 \mathrm{~Hz}$, referenced to $\mathrm{Cz}$. As suggested for the EGI high input impedance amplifier, impedances were kept below $50 \mathrm{k} \Omega$. All channels were preprocessed on-line by means of $0.1-200 \mathrm{~Hz}$ band-pass filtering.

\subsection{Data reduction and analysis}

EEG was segmented response-locked to obtain epochs containing $2000 \mathrm{~ms}$ prior to and $1000 \mathrm{~ms}$ following the button press. Only rewarded epochs (i.e. 30 points and higher) were included both for the random and the shaping condition in order to exclude trials with multiple key presses and those that reflected erratic key pressing. Artifact correction was performed by means of the 'statistical correction of artifacts in dense array studies' procedure developed by Junghöfer et al. [19]. This method uses a combination of trial exclusion and channel approximation based on statistical parameters of the data. In a first step, this procedure detects recording channel artifacts using the recording reference (i.e. Cz). Subsequently, global artifacts are detected using the average reference. In a next interactive step, electrodes from particular trials are removed on the basis of the distribution of their amplitude, standard deviation and gradient. The information of eliminated electrodes is replaced with a statistically weighted spherical interpolation from the full channel set. In a last step, the variance of the signal across trials is computed to document the stability of the average waveform. The limit for the number of approximated channels was set to 20 channels. With respect to the spatial arrangement of the approximated electrodes, it was ensured that the rejected channels were not located within one region of the scalp, because this would make interpolation for this area invalid. To this end, the standard deviation of the spherical splines used for approximation was computed for each trial and trials that represented outliers from this distribution were rejected [19]. Single epochs with excessive eye-movements and blinks or more than 20 channels containing artifacts were discarded. After artifact correction, the mean number of trials remaining across conditions was 69 , with a minimum value of 59 and a maximum value of 92 artifact-free trials. No significant differences of trial numbers emerged between the random reinforcement (mean number of artifact-free trials in analysis: 62) and the shaping condition (mean number of artifact-free trials in analysis: 67). The self-paced condition however contained significantly more artifact free trials than the random and 
the shaping condition (mean: 82 trials, ANOVA, $P<0.05$ ). Prior to further analyses, artifact free EEG epochs were algebraically re-referenced to the average reference.

\subsubsection{Data analysis $G B A$}

Transformation of artifact free EEG epochs into the frequency domain was obtained by convolution with complex wavelets. Complex Morlet wavelets were used to overcome problems with a constant Fourier window length. This procedure has the advantage that the time resolution for high frequencies is better compared to low frequency ranges, where frequency resolution is better, but time resolution is coarse. Thus, this technique is especially suited for detecting induced high-frequency oscillations that may occur during brief periods of time. The present procedure has been proposed by Bertrand et al. [3] and is described in detail in the respective publications [48,49]. In brief, complex Morlet wavelets $g$ can be generated in the time domain for different analysis frequencies $f_{0}$ according to

$g\left(t, f_{0}\right)=A^{\prime} e^{\frac{t^{2}}{2 \sigma_{t}^{2}}} e^{2 \iota \pi f_{0} t}$

with $A^{\prime}$ depending on the parameter $\sigma_{f}$, specifying the width of the wavelet in the frequency domain, the analysis frequency $f_{0}$ and the user-selected ratio $m$ :

$A^{\prime}=\sigma_{f} \sqrt{2 \pi^{3}} \sqrt{\frac{m}{f_{0} \sqrt{\pi}}}$

with

$m=\frac{f_{0}}{\sigma_{f}}$

Thus, given a constant ratio $m$, the width of the wavelets in the frequency domain, $\sigma_{f}$, changes as a function of the analysis frequency $f_{0}$. A constant $m=f_{0} / \sigma_{f}=7$ was select- ed in order to achieve good time and frequency resolution for the examined frequency range from 9.8 to $61.5 \mathrm{~Hz}$. Wavelets of this family were normalized in order to have equal amounts of energy. For each artifact-free epoch, time-varying energy in a given frequency band was obtained as the squared absolute value of the convolution of the cosine-square tapered signal with the wavelet. Single trial time by frequency matrices were averaged across recording epochs, response-locked to the button press that simultaneously elicited feedback. Thus the response-locked evolutionary spectrum was obtained for each electrode and condition. A 1900-1750-ms period prior to the button press was used as baseline for the time-frequency information, in order to avoid confound with motor preparation and execution, as well as movement artifacts. The mean of this time window was subtracted from the time-frequency matrix for each frequency and time point.

Spectral power in five frequency bands was obtained by computing the power for alpha $(10-13 \mathrm{~Hz})$, beta (14-20 $\mathrm{Hz})$, low gamma $(21-30 \mathrm{~Hz})$, mid gamma $(31-40 \mathrm{~Hz})$, and high gamma (40-62 Hz). The three frequency bands in the gamma range were analyzed separately because they differed in time-course and topography. In particular, the dissociation of the $21-30 \mathrm{~Hz}$ band from both beta and higher gamma bands at anterior sites appeared to justify separation of the three gamma bands (see Figs. 2 and 6). All frequency bands were examined in five time windows: (1) 400-200 ms prior to the button press, (2) 180-250, (3) 250-350, (4) 350-500, and (5) 500-650 ms following the button press. Since the focus of the present work was on high-frequency oscillations, the time windows were selected on the basis of previous findings regarding (a) timing of visual GBA modulations [47] (windows 3 and 4), (b) movement-related GBA modulations [36,37] (window 1), and (c) based on grand mean time-frequency information

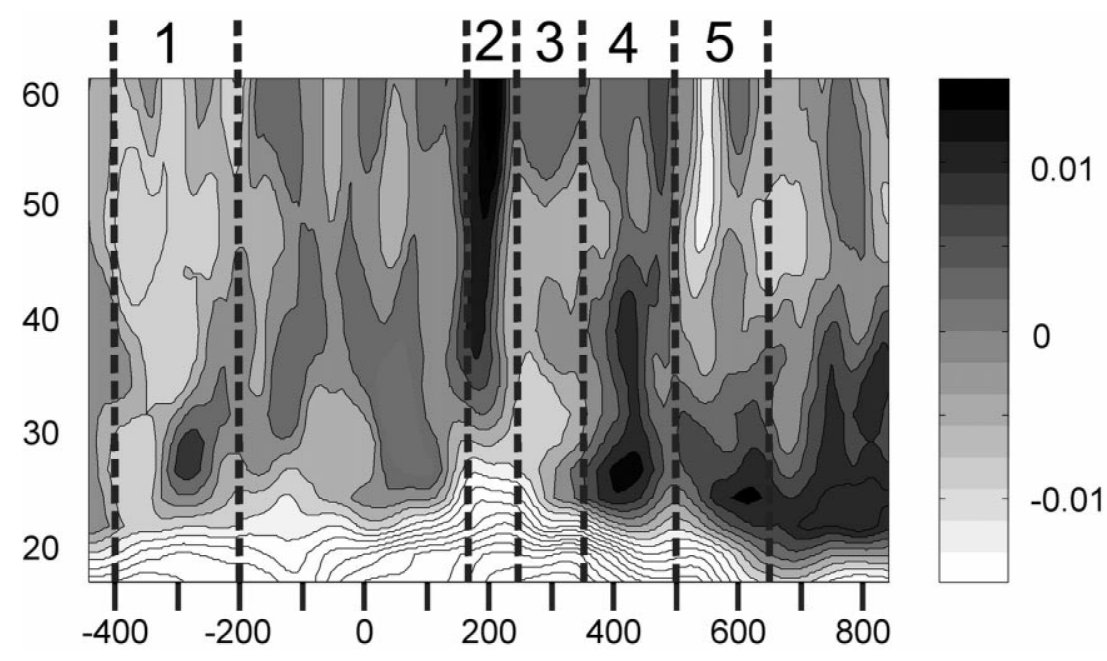

Fig. 2. Grand mean $(n=11)$ evolutionary spectrum across the three experimental conditions (shaping, random reward, self-paced pressing), averaged across electrodes included in the statistical analyses. Numbers at the top of the figure indicate the time windows selected for statistical analyses. 
shown in Fig. 2. The same time windows were examined for the lower bands (i.e. alpha and beta) to control for effects of higher harmonics. In order to assess statistically the effects of experimental conditions on frontal and temporo-occipital oscillatory activity, spectral power at the 129 electrodes was grouped into four regional means covering fronto-central and occipito-temporal sites. These regions have been shown sensitive to GBA modulations in studies using identical electrode montages [21,28]. Each region comprised 12 electrodes. Fig. 3 displays their relative position with respect to sites of the international 10-20 system.

For each time window and frequency range, mean spectral power in these regions was subject to a threefactor ANOVA with the within-subjects factors Condition (shaping, random, self-paced), Hemisphere (left/right), and Region (anterior/posterior). As a control, frequency measures in the baseline interval (1900-1750 ms prior to the button press) were also subject to the same ANOVAs. Degrees of freedom were adjusted by means of the Greenhouse-Geisser method where appropriate [12]. Corrected $P$ values are reported. Post-hoc comparisons were evaluated by means of the Scheffé test.

\subsubsection{Analysis of behavioral data}

Two variables were used to examine effects of shaping in the present study: (1) the mean amount of reward obtained in the shaping and the random reinforcement condition, and (2) the difference between the mean response latency and the ideal maximum reward latency of $3000 \mathrm{~ms}$. Effects of shaping versus random reinforcement

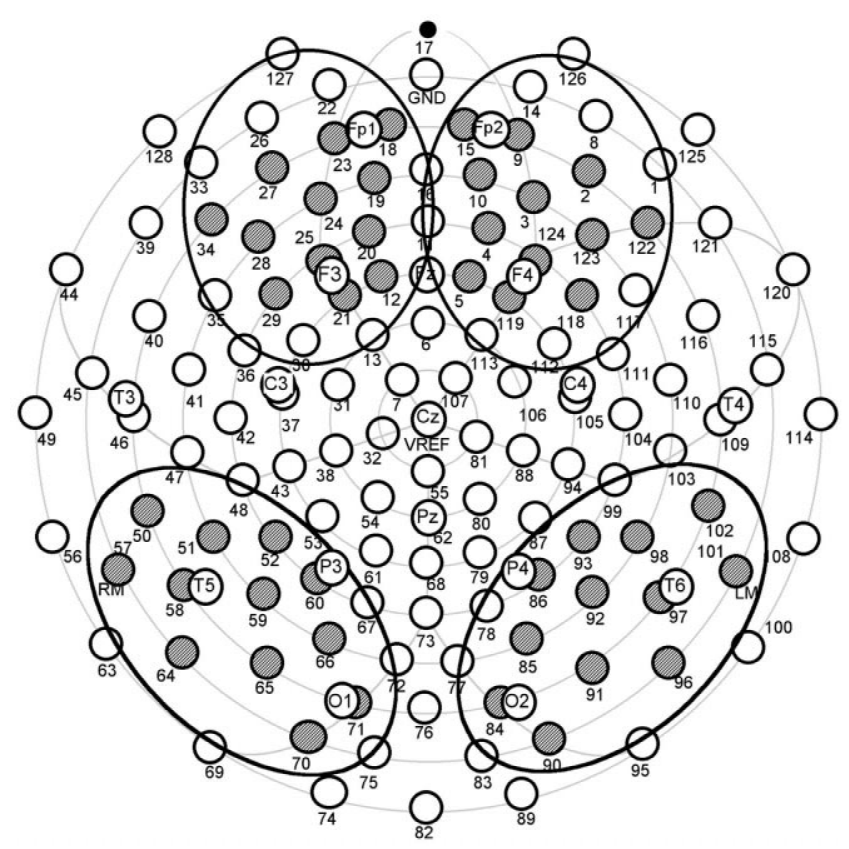

Fig. 3. Electrode groups formed for statistical testing. Encircled sites were used for regional means of spectral measures as tested in ANOVAs. on these two measures were evaluated using paired $t$-tests. Data from aborted trials were excluded from these analyses.

\section{Results}

\subsection{Behavioral results}

The shaping procedure was successful in establishing a motor response in the required time window (see Fig. 4). As expected, the mean reward across trials was significantly higher in the shaping than in the random condition $(t(10)=-14.2 ; P<0.01)$. Likewise, the distance between the actual response delays and the maximum reward delay was smaller during shaping $(t(10=4.7 ; P<0.01)$ than during random reward.

\subsection{Spectral measures}

\subsubsection{Time windows preceding the button press}

In the baseline time window, differences between conditions in the beta and gamma bands did not reach statistical significance. There was a trend towards greater posterior alpha reduction in the shaping condition, compared to the self-paced and random reinforcement conditions [Condition $\times$ Region $(F(2,20)=3.2 ; P<0.09$ ].

In the time window $400-200 \mathrm{~ms}$ prior to the button press, alpha reduction was significantly greater for the shaping and self-paced condition, compared to random reinforcement (Condition; $F(2,20)=8.0 ; P<0.01$; Scheffé $P$ s $<0.05)$.

No further significant effects of experimental conditions on spectral measures were found in the time window preceding the button press the presentation of the feedback stimulus. However, this time window showed a broadly distributed enhancement of the mid gamma frequency $(31-40 \mathrm{~Hz})$ power at anterior sites, which resulted in a significant main effect of Region, indicating greater power at anterior than posterior sites across conditions $(F(1,10)=$ $7.1 ; P<0.05)$. The remaining bands did not show topographical differences in this time range.

\subsubsection{Time windows following the button press}

3.2.2.1. Alpha band (10-13 Hz). ANOVAs on the mean spectral alpha power showed that throughout time windows, significant effects of Region indicated that alpha reduction was more pronounced at posterior, compared to anterior sites $(F \mathrm{~s}(1,10)=5.9,7.4,5.6,6.1 ; P<0.05$, respectively). Additionally, the left hemisphere showed greater alpha reduction in the first time window (180-250 


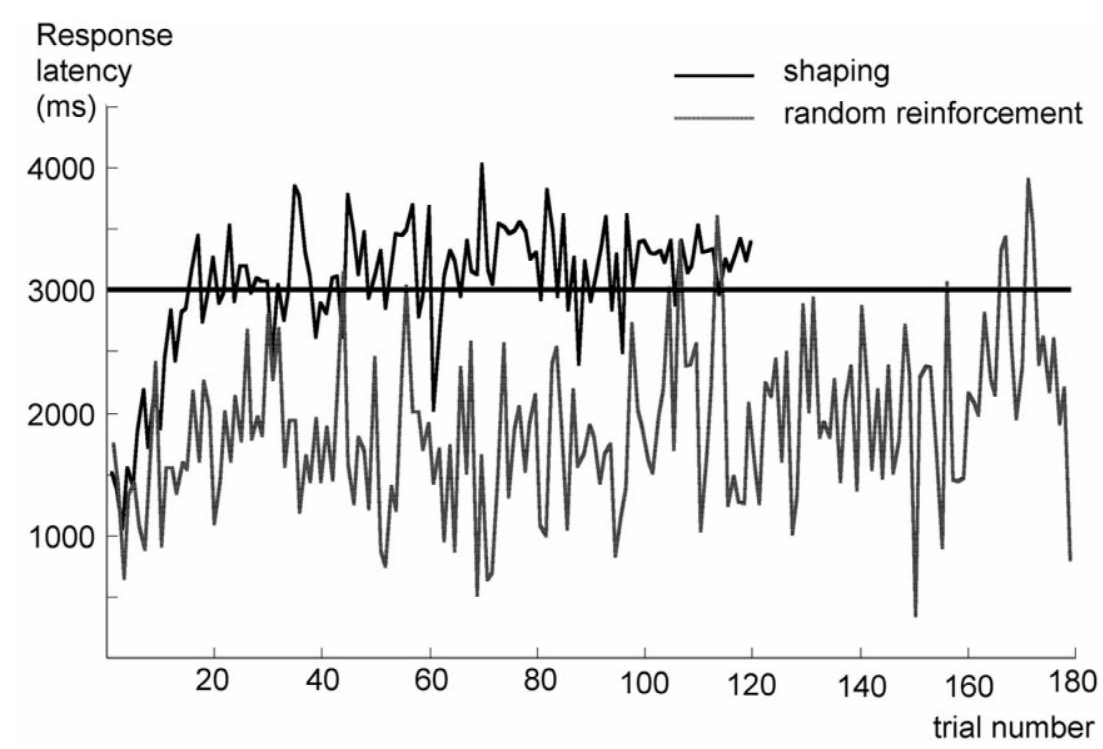

Fig. 4. Grand mean latencies of key presses with respect to the onset cue (color change of fixation cross) for the shaping condition (black line, 120 trials) and the random reinforcement condition (dashed line, 180 trials). Note that the shaping regime was effective in enhancing likelihood of response latencies near the lower boundary of the limited hold interval, i.e. $3000 \mathrm{~ms}$ after the cue (thick horizontal line).

ms, $F(1,10)=15.7 ; P<0.01)$. No further effects were seen in this frequency range, including no effects of Condition.

3.2.2.2. Beta band (14-20 Hz). Similarly to alpha, beta displayed a decrease with respect to baseline level throughout time windows. This decrease was more pronounced at posterior than anterior sites in the three later time segments (i.e. $250-350,350-500$, and 500-650 ms; Region: $F \mathrm{~s}(1,10)=11.0,15.5,10.8 ; P<0.01)$. Furthermore, beta decrease at $250-350 \mathrm{~ms}$ discriminated between experimental conditions (Condition: $F(2,20)=6.8 ; P<0.01$ ), with greater beta reduction in the shaping condition compared to both control conditions (Scheffé $P<0.01$ ). A significant interaction Condition $\times$ Region $(F(2,20)=3.7 ; P<0.05)$ demonstrated that posterior recording sites contributed most to this difference (Scheffé $P \mathrm{~s}<0.05$, see Fig. 5). A similar picture emerged for the $350-500 \mathrm{~ms}$ window. Again, beta decrease was greater during shaping than during random reinforcement and self-paced pressing (Condition: $F(2,20)=5.1 ; P<0.05$; Scheffé $P \mathrm{~s}<0.05$ ), and this effect was significantly greater at posterior regions (Condition $\times$ Region: $F(2,20)=5.8 ; P<0.05$ ). No effects involving Condition were found in the late time window (500-650 ms)

3.2.2.3. Low gamma band $(21-30 \mathrm{~Hz})$. There was no significant main effect or interaction in the 180-250-ms window. However, in the $250-350-\mathrm{ms}$ range, a prominent decrease of low gamma power was seen at posterior sites $(F(1,10)=10.8 ; P<0.01)$ that was most pronounced for the shaping condition across hemispheres (Condition $\times$ Region: $F(2,20)=3.8 ; P<0.05)$. No effects of Condition were seen at anterior sensors in this time range. In contrast, the 350-500 and 500-650-ms window exhibited patterns showing pronounced anterior differences between experimental conditions. Between 350 and $500 \mathrm{~ms}$, a Condition $\times$ Region interaction $(F(2,20)=7.1 ; \quad P<0.05)$ showed that low gamma power during shaping was significantly enhanced at anterior electrodes, compared to control conditions (Scheffé $P \mathrm{~s}<0.05$ ). Additionally, an effect of Region $(F(1,10)=18.8 ; P<0.01)$ indicated an increase at anterior sites and a decrease at posterior sites, across conditions.

Similar effects were observed in the late time window (500-650 ms). However, these effects showed a differential hemispheric preponderance. Again, the significant Condition $\times$ Region interaction $(F(2,20)=4.8 ; P<0.05$; see Fig. 6) revealed power enhancement at anterior sites for the shaping condition (Scheffé $P \mathrm{~s}<0.05$ ). Low gamma power was significantly enhanced at left anterior sensors under the shaping regime, whereas both control conditions did not show such an enhancement (Condition $\times$ Region $\times$ Hemisphere: $F(2,20)=10.1 ; P<0.01)$. This effect is illustrated in Fig. 7.

3.2.2.4. Mid gamma band (31-40 $\mathrm{Hz})$. A significant interaction Condition $\times$ Region $(F(2,20)=3.7 ; \quad P<0.05)$ was observed in the 180-250-ms time window, demonstrating that spectral power during shaping and random reward was higher at posterior than anterior sensors across hemispheres (Scheffé $P<0.05$ ). No such topographical difference was observed for the self-paced condition, where no visual feedback stimulus was given. However, this effect was restricted to this time range. Testing later 

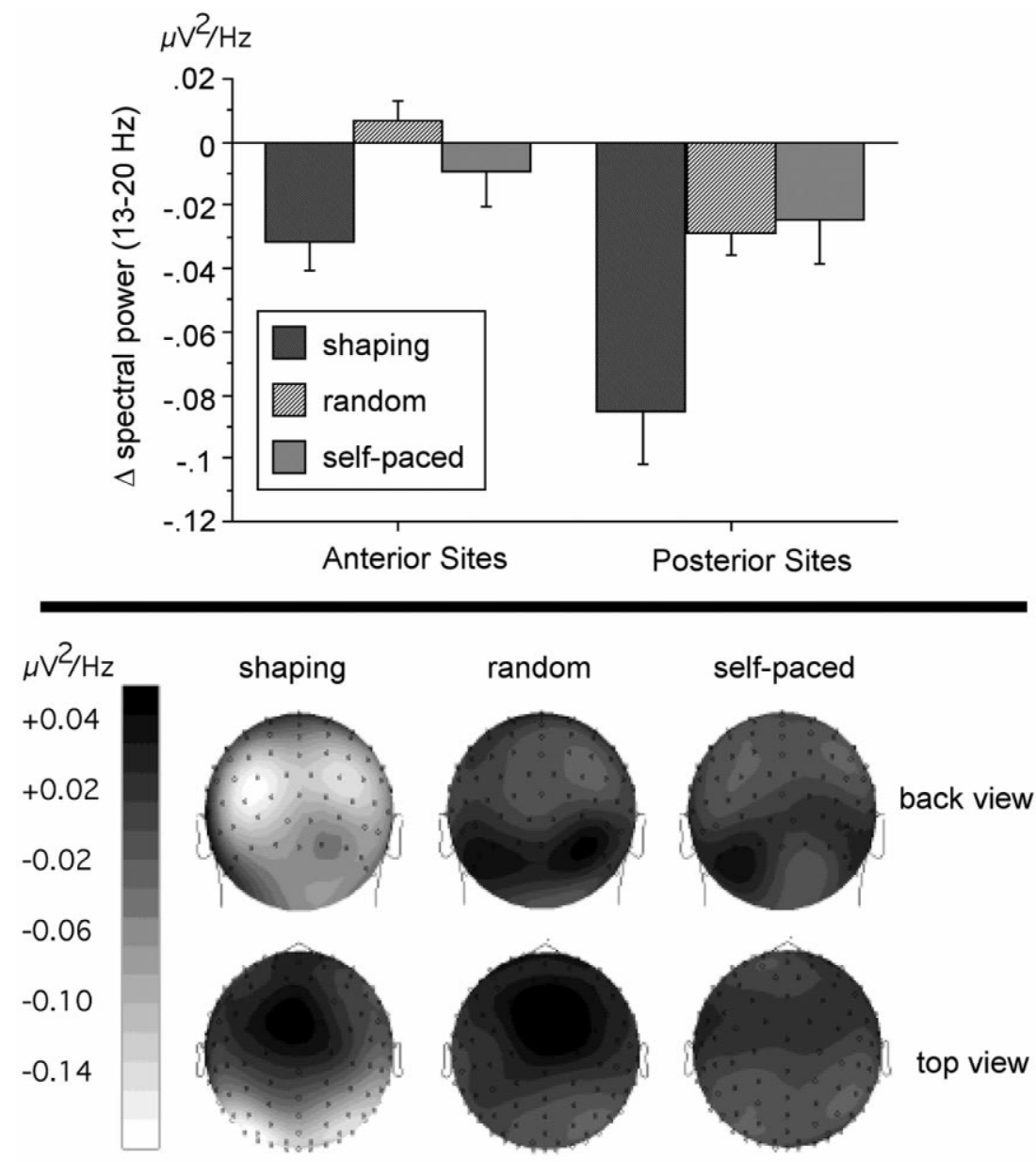

Fig. 5. Mean changes of spectral power with respect to baseline in the beta band (13-20 Hz) in the 250-350-ms time window, displayed separately for anterior/posterior recording sites and experimental condition. Values represent a mean of $n=11$ subjects. Bars indicate standard error. Bottom panel: topographical distribution of the spectral power in this time-frequency window.

time windows revealed no significant effects of Condition on mid gamma power.

3.2.2.5. High gamma band (41-62 Hz). A similar picture emerged for the high frequency range during the early window (Condition $\times$ Region: $\quad F(2,20)=3.7 ; \quad P<0.05$ ). Again, there was a specific anterior-posterior difference during shaping and random reward only $(P<0.05)$, with posterior sensors exhibiting higher spectral power than anterior sensors. Subsequent time ranges showed no significant effects involving Condition.

\section{Discussion}

Using an operant learning paradigm involving shaping of a time-dependent motor response, we found that oscillatory activity differentiated between the processing of reward stimuli (i.e. display of money gained) while contingent as compared to non-contingent reinforcement was presented to the participants. Lower gamma band activity showed a frontal increase starting at about $400 \mathrm{~ms}$ after the presentation of response-contingent reward (see Fig. 6). Interestingly, this effect was weaker, but also present in the control condition that required repetitive self-paced key presses without any feedback. One explanation for this finding may lie in the fact that both conditions involved planning of the subsequent response after termination of the key press. However, the low gamma modulation obtained during self-paced pressing was (1) attenuated compared to the shaping condition and (2) showed a different topographical distribution, with a maximum on right frontal sites. Thus, it cannot be assumed that motor planning of the subsequent response alone accounts for the effects observed in the current study. Rather, the left frontal power change observed during shaping seems to be specific for processing contingencies between behaviors and external stimuli with a behavioral or motivational relevance, i.e. reinforcers. The latter aspect was not present during random reinforcement, when reward was unpredictable and participants were unable to learn a contingent response or to plan subsequent motor behavior. According- 

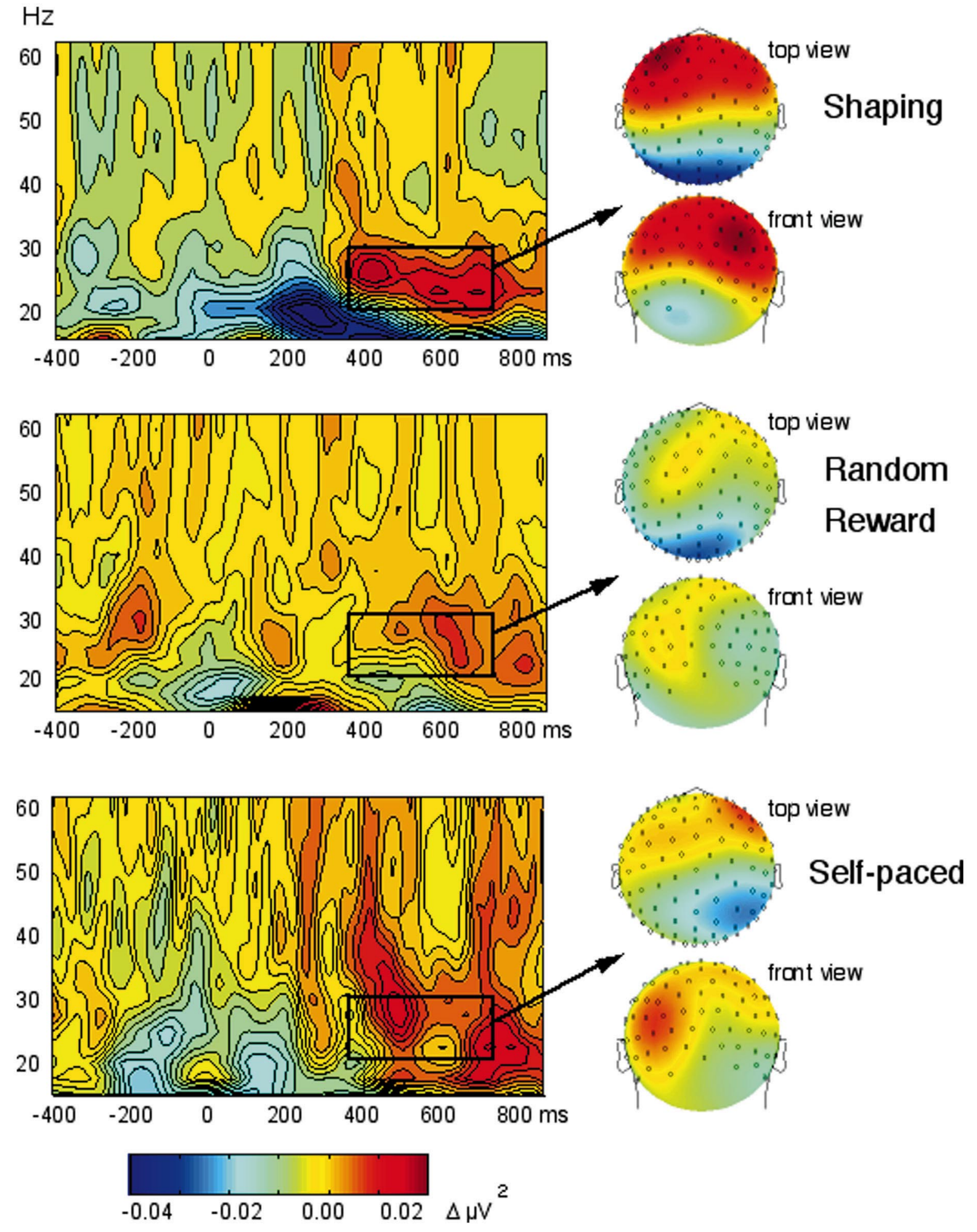

Fig. 6. Left: grand mean time-frequency plots for three experimental conditions - shaping, random reward, self-paced pressing. Plots reflect an aver age across electrodes anterior to Cz. A baseline mean $(-1900$ to $-1750 \mathrm{~ms})$ was subtracted for each frequency band. Time-point 0 corresponds to the button press and onset of feedback stimulus. Right: topographical distribution of the spectral power in the time-frequency window outlined in the time-frequency plots. 


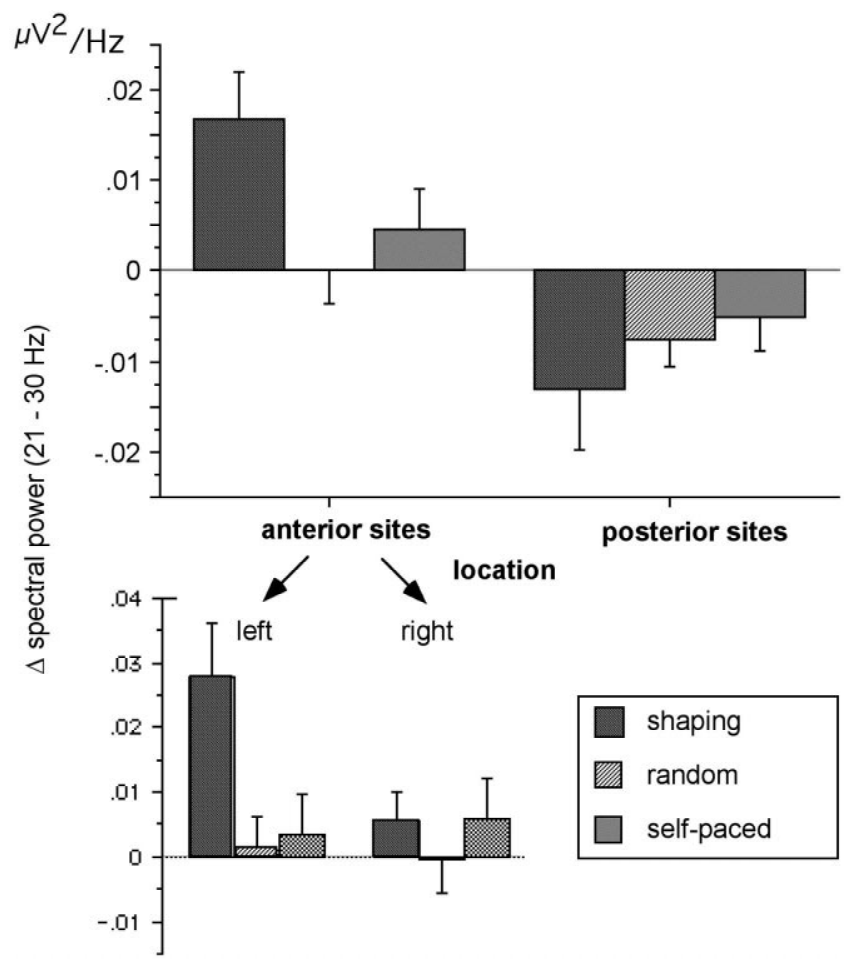

Fig. 7. Mean changes of spectral power with respect to baseline in the lower gamma band $(20-30 \mathrm{~Hz})$ in the $550-650-\mathrm{ms}$ time window. Values represent a mean of $n=11$ subjects. Bars indicate standard error.

ly, frontal low gamma modulation was weakest in this condition. Thus, a dissociation of motor planning and processing of task-relevant external information is suggested by the different topographical distributions. This interpretation is consistent with the view that areas in prefrontal cortex are elements of networks necessary for assembling memories and behavioral patterns that enable the individual to react appropriately on the basis of past experience [54]. In addition, it is in line with recent reports from neuroimaging studies showing left prefrontal activation during associative learning $[27,53]$.

Alternative views are based on findings of human EEG coherency in the gamma range. For example, Miltner et al. [26] reported an increase in cross-electrode site synchrony during classical conditioning in human participants that was restricted to the mid gamma frequency range around $40 \mathrm{~Hz}$, but did not observe power modulation in this band. In the present study, power changes of anterior low GBA were seen in a band well below the $37-43 \mathrm{~Hz}$ range where phase synchrony effects were most pronounced in the Miltner et al. study [26]. Thus, different frequency bands may provide complementary information in terms of changes in power/amplitude, inter-site synchrony, time course, or topographical distribution. In line with this view, Rodriguez et al. [40] showed that power modulation and changes of phase-synchrony in a single frequency band might occur in different time windows during visual object recognition. These authors have proposed that specific inter-site synchrony without GBA amplitude enhancement may be viewed as a mechanism of large-scale cognitive integration, which is also necessary during the learning process, whereas GBA amplitude modulation would reflect changes in local oscillatory processes. One possible correlate of different levels of integration is the temporal frequency of the respective large-scale oscillation. For example, von Stein and Sarnthein [43,51,52], have argued that synchronization at different frequencies of large-scale brain electric activity might be used for integration of cell assemblies being involved in tasks differing in complexity. Thus, less complex unimodal perceptual processes are suggested to involve high-frequency oscillations, whereas more complex multimodal processing should relate to modulations in lower bands such as theta or alpha. While the present work did not examine inter-site coherence, the dissociation between (1) posterior modulations of mid gamma power $(31-40 \mathrm{~Hz})$ in the $180-250-\mathrm{ms}$ time windows and (2) anterior modulations of low gamma $(21-30 \mathrm{~Hz})$ in the late time window (500-650 ms) would be consistent with this suggestion.

The anterior GBA $(31-40 \mathrm{~Hz})$ enhancement prior to movement found in the current study represents a replication of results reported by several authors $[11,20,22,46]$. This effect seems to be related to motor preparation/ execution and may be an oscillatory counterpart to the readiness potential. We extended these studies by showing that this response is not modulated by the motivational relevance of the motor response. The fact that we did not find GBA differences between conditions in the time window preceding the button press may be interpreted as evidence against task-specific large-scale high-frequency activity prior to the operant behavior. However, highfrequency concomitants of movement were clearly not the focus of the present study. Given the broad anterior distribution of the $20-30 \mathrm{~Hz}$ enhancement during shaping, it is possible that pre-motor areas are involved in processing information that serves to adjust behavior to the changing requirements of a reinforcement schedule. Similar phenomena have been reported with respect to oscillatory activity in this frequency range during execution of motor tasks in monkeys [29-31]. Interestingly, these oscillations have been observed in the lower gamma range, paralleling the present findings. Future work may assess the question of cortical sources of oscillatory brain responses using distributed source modeling of single sweep EEG epochs before transformation into frequency-domain measures [14,15].

Regarding the alpha and beta bands, we replicated the pre-movement alpha power reduction that has been described repeatedly by Pfurtscheller and coworkers [34,35] and has been referred to as event-related alpha desynchronization. Interestingly, this effect showed sensitivity to experimental conditions, with random reinforcement being related to less alpha desynchronization than shaping or self-paced key pressing. This finding may reflect the fact 
that movement preparation was possible in the latter conditions, but was more difficult when contingencies were unclear. Moreover, the shaping condition was specifically characterized by a decrease in the beta band at posterior sensors. This appears to be consistent with reports of a correlation between beta activity and allocation of processing resources for cognitive processes in the visual modality [39]. While no visual feedback was provided in the self-paced condition, the shaping and the random reinforcement conditions differed in that they involved predictable (shaping) or non-predictable (random reinforcement) visual feedback, thus requiring different amounts of processing resources.

Interestingly, the GBA enhancement around $40 \mathrm{~Hz}$ that has regularly been observed at posterior electrodes in time segments ranging from 200 to $400 \mathrm{~ms}$ after the onset of a visual stimulus [47], was weak in the present study. However, in the mid and high gamma range it reached significance when comparing shaping and random reinforcement with self-paced button pressing, where no visual stimulus was displayed. This result of reduced activity in visual areas is in line with reports from neuroimaging experiments of learning. For example, Desimone [7] has proposed a model that suggests suppression of activity in visual cortex as a consequence of repetitive stimulus presentation during learning. The present data suggest that the induced GBA in the classical gamma range around 40 $\mathrm{Hz}$ reflect these processes, whereas lower GBA in the present design does not show such alterations. Furthermore, activity in frontal-temporal loops is assumed to moderate learning and memory as well as direct spatial selective attention to motivationally relevant objects in the field of view [7]. Both the present data as well as findings reported by Tallon-Baudry et al. [40] are consistent with this view. The latter authors found frontal and occipitotemporal GBA enhancement in the $20-30 \mathrm{~Hz}$ range during the delay phase of a delayed matching-to-sample task. This power enhancement however showed a different topographical distribution of the task-related GBA modulation, compared to the present study. This seems plausible because both the present task and the delayed matching-tosample paradigm involve memory formation, but the present design emphasized operant learning and behavior modification rather than activation of a visual object's mental representation.

In summary, the present data have several implications for understanding the macroscopic neural mechanisms of learning. We found temporally extended anterior low gamma activity in a late time window ( $>400 \mathrm{~ms}$ ), as could be predicted by theoretical accounts of prefrontal contribution to operant learning [5,41]. The time course of activation as found in the present study supports the notion that besides reflecting visual processing, induced high-frequency oscillatory activity might also be a correlate of the higher-order cognitive processes following perceptual integration. The present results can be regarded supportive for the view that acquisition of new behaviors is embodied in variable networks differing in distribution and elements, depending on the internal processing steps performed in response to a stimulus and on the nature of external contingencies.

\section{Acknowledgements}

The authors would like to thank Edward Taub for helpful discussions and Ursula Lommen and Heidi Messmer for help in data acquisition. This work was supported by the Deutsche Forschungsgemeinschaft.

\section{References}

[1] E. Basar, C. Basar-Eroglu, S. Karakas, M. Schurmann, Brain oscillations in perception and memory, Int. J. Psychophysiol. 35 (2000) 95-124.

[2] G.C. Baylis, E.T. Rolls, Responses of neurons in the inferior temporal cortex in short term and serial recognition memory tasks, Exp. Brain Res. 65 (1987) 614-622.

[3] O. Bertrand, J. Bohorquez, J. Pernier, Time-frequency digital filtering based on an invertible wavelet transform: an application to evoked potentials, IEEE Trans. Biomed. Eng. 41 (1994) 77-88.

[4] C. Buchel, J.T. Coull, K.J. Friston, The predictive value of changes in effective connectivity for human learning, Science 283 (1999) $1538-1541$.

[5] A.R. Damasio, On some functions of the human prefrontal cortex, Ann. NY Acad. Sci. 769 (1995) 241-251.

[6] E.L. Deci, R. Koestner, R.M. Ryan, A meta-analytic review of experiments examining the effects of extrinsic rewards on intrinsic motivation, Psychol. Bull. 125 (1999) 627-668, discussion pp. 692-700.

[7] R. Desimone, Neural mechanisms for visual memory and their role in attention, Proc. Natl. Acad. Sci. USA 93 (1996) 13494-13499.

[8] V.N. Dumenko, Dynamic shifts in the parameters of the traditional frequency range of the EEG during learning in dogs, Neurosci. Behav. Physiol. 25 (1995) 403-412.

[9] R. Eckhorn, A. Obermueller, Single neurons are differently involved in stimulus-specific oscillations in cat visual cortex, Exp. Brain Res. 95 (1993) 177-182.

[10] W.J. Freeman, Role of chaotic dynamics in neural plasticity, Prog. Brain Res. 102 (1994) 319-333.

[11] C. Gerloff, J. Richard, J. Hadley, A.E. Schulman, M. Honda, M. Hallett, Functional coupling and regional activation of human cortical motor areas during simple, internally paced and externally paced finger movements, Brain 121 (1998) 1513-1531.

[12] S.W. Greenhouse, S. Geisser, On methods in the analysis of profile data, Psychometrika 24 (1959) 95-112.

[13] E. Halgren, K. Marinkovitch, Neurophysiological networks integrating human emotions, in: M.S. Gazzaniga (Ed.), The Cognitive Neurosciences, MIT Press, Cambridge, MA, 1995, pp. 1137-1151.

[14] M. Hämäläinen, R. Ilmoniemi, Interpreting Measured Magnetic Fields of the Brain: Estimates of of Current Distributions, Helsinki University of Technology, Helsinki, 1984.

[15] O. Hauk, P. Berg, C. Wienbruch, B. Rockstroh, T. Elbert, The minimum norm method as an effective mapping tool for MEG analysis, in: Biomag98, Sendai, Japan, 1998.

[16] D. Hebb, The Organization of Behavior; A Neuropsychological Theory, Wiley, New York, 1949.

[17] O. Jensen, J.E. Lisman, Theta/gamma networks with slow NMDA 
channels learn sequences and encode episodic memory: role of NMDA channels in recall, Learn. Mem. 3 (1996) 264-278.

[18] V.K. Jirsa, R. Friedrich, H. Haken, J.A. Kelso, A theoretical model of phase transitions in the human brain, Biol. Cybern. 71 (1994) $27-35$.

[19] M. Junghöfer, T. Elbert, D.M. Tucker, B. Rockstroh, Statistical control of artifacts in dense array EEG/MEG studies, Psychophysiology 37 (2000) 523-532.

[20] J. Kaiser, W. Lutzenberger, H. Preissl, D. Mosshammer, N. Birbaumer, Statistical probability mapping reveals high-frequency magnetoencephalographic activity in supplementary motor area during self-paced finger movements, Neurosci. Lett. 283 (2000) $81-84$.

[21] A. Keil, M.M. Muller, W.J. Ray, T. Gruber, T. Elbert, Human gamma band activity and perception of a gestalt, J. Neurosci. 19 (1999) 7152-7161

[22] R. Kristeva-Feige, B. Feige, S. Makeig, B. Ross, T. Elbert, Oscillatory brain activity during a motor task, NeuroReport 4 (1993) 1291-1294.

[23] J.E. LeDoux, Emotional networks in the brain, in: J.M.H. Michael Lewis (Ed.), Handbook of Emotions, Guilford Press, New York, 1993, pp. 109-118.

[24] W. Lutzenberger, H. Preissl, N. Birbaumer, F. Pulvermuller, Highfrequency cortical responses: do they not exist if they are small?, Electroencephalogr. Clin. Neurophysiol. 102 (1997) 64-66.

[25] P.M. Milner, A model for visual shape recognition, Psychol. Rev. 81 (1974) 521-535.

[26] W.H. Miltner, C. Braun, M. Arnold, H. Witte, E. Taub, Coherence of gamma-band EEG activity as a basis for associative learning, Nature 397 (1999) 434-436.

[27] S.E. Molchan, T. Sunderland, A.R. McIntosh, P. Herscovitch, B.G. Schreurs, A functional anatomical study of associative learning in humans, Proc. Natl. Acad. Sci. USA 91 (1994) 8122-8126.

[28] M.M. Müller, A. Keil, T. Gruber, T. Elbert, Processing of affective pictures modulates right-hemispheric gamma band EEG activity, Clin. Neurophysiol. 110 (1999) 1913-1920.

[29] V.N. Murthy, E.E. Fetz, Coherent 25- to $35-\mathrm{Hz}$ oscillations in the sensorimotor cortex of awake behaving monkeys, Proc. Natl. Acad. Sci. USA 89 (1992) 5670-5674.

[30] V.N. Murthy, E.E. Fetz, Oscillatory activity in sensorimotor cortex of awake monkeys: synchronization of local field potentials and relation to behavior, J. Neurophysiol. 76 (1996) 3949-3967.

[31] V.N. Murthy, E.E. Fetz, Synchronization of neurons during local field potential oscillations in sensorimotor cortex of awake monkeys, J. Neurophysiol. 76 (1996) 3968-3982.

[32] A.J. Nonneman, J.V. Corwin, Differential effects of prefrontal cortex ablation in neonatal, juvenile, and young adult rats, J. Comp. Physiol. Psychol. 95 (1981) 588-602.

[33] R. Numan, K.A. Grant, Lateral, but not medial, frontal lesions impair fixed ratio performance in rats, Physiol. Behav. 24 (1980) $625-627$.

[34] G. Pfurtscheller, A. Aranibar, Evaluation of event-related desynchronization (ERD) preceding and following voluntary selfpaced movement, Electroencephalogr. Clin. Neurophysiol. 46 (1979) $138-146$.

[35] G. Pfurtscheller, A. Berghold, Patterns of cortical activation during planning of voluntary movement, Electroencephalogr. Clin. Neurophysiol. 72 (1989) 250-258.
[36] G. Pfurtscheller, C. Neuper, Simultaneous EEG $10 \mathrm{~Hz}$ desynchronization and $40 \mathrm{~Hz}$ synchronization during finger movements, NeuroReport 3 (1992) 1057-1060.

[37] G. Pfurtscheller, C. Neuper, J. Kalcher, 40-Hz oscillations during motor behavior in man, Neurosci. Lett. 164 (1993) 179-182.

[38] F. Pulvermüller, A. Keil, T. Elbert, High-frequency brain activity: perception or active memory ?, Trends Cogn. Sci. 3 (1999) 250253.

[39] W.J. Ray, H.W. Cole, EEG alpha activity reflects attentional demands, and beta activity reflects emotional and cognitive processes, Science 228 (1985) 750-752.

[40] E. Rodriguez, N. George, J.P. Lachaux, J. Martinerie, B. Renault, F.J. Varela, Perception's shadow: long-distance synchronization of human brain activity [In Process Citation], Nature 397 (1999) 430-433.

[41] E.T. Rolls, A theory of emotion, and its application to understanding the neural basis of emotion, Cogn. Emotion 4 (1990) 161-190.

[42] E.T. Rolls, H.D. Critchley, R. Mason, E.A. Wakeman, Orbitofrontal cortex neurons: role in olfactory and visual association learning, J. Neurophysiol. 75 (1996) 1970-1981.

[43] J. Sarnthein, H. Petsche, P. Rappelsberger, G.L. Shaw, A. von Stein, Synchronization between prefrontal and posterior association cortex during human working memory, Proc. Natl. Acad. Sci. USA 95 (1998) 7092-7096.

[44] D.R. Shanks, Human instrumental learning: a critical review of data and theory, Br. J. Psychol. 84 (1993) 319-354.

[45] W. Singer, C.M. Gray, Visual feature integration and the temporal correlation hypothesis, Annu. Rev. Neurosci. 18 (1995) 555-586.

[46] S. Slobounov, R. Simon, R. Tutwiler, M. Rearick, EEG correlates of wrist kinematics as revealed by averaging techniques and Morlet wavelet transforms, Motor Control 4 (2000) 350-372.

[47] C. Tallon-Baudry, O. Bertrand, Oscillatory gamma activity in humans and its role in object representation, Trends Cogn. Sci. 3 (1999) 151-162.

[48] C. Tallon-Baudry, O. Bertrand, C. Delpuech, J. Pernier, Oscillatory gamma-band $(30-70 \mathrm{~Hz})$ activity induced by a visual search task in humans, J. Neurosci. 17 (1997) 722-734.

[49] C. Tallon-Baudry, O. Bertrand, F. Peronnet, J. Pernier, Induced gamma-band activity during the delay of a visual short-term memory task in humans, J. Neurosci. 18 (1998) 4244-4254.

[50] C. von der Malsburg, J. Buhmann, Sensory segmentation with coupled neural oscillators, Biol. Cybern. 67 (1992) 233-242.

[51] A. von Stein, P. Rappelsberger, J. Sarnthein, H. Petsche, Synchronization between temporal and parietal cortex during multimodal object processing in man, Cereb. Cortex 9 (1999) 137-150.

[52] A. von Stein, J. Sarnthein, Different frequencies for different scales of cortical integration: from local gamma to long range alpha/theta synchronization, Int. J. Psychophysiol. 38 (2000) 301-313.

[53] A.D. Wagner, R.A. Poldrack, L.L. Eldridge, J.E. Desmond, G.H. Glover, J.D. Gabrieli, Material-specific lateralization of prefrontal activation during episodic encoding and retrieval, NeuroReport 9 (1998) 3711-3717.

[54] D.H. Zald, S.W. Kim, Anatomy and function of the orbital frontal cortex. II. Function and relevance to obsessive-compulsive disorder, J. Neuropsychiatry Clin. Neurosci. 8 (1996) 249-261. 